\title{
MHD relaxation of fossil magnetic fields in stellar interiors
}

\author{
Stéphane Mathis ${ }^{1,2}$, Vincent Duez ${ }^{3}$ \& Jonathan Braithwaite ${ }^{3}$ \\ ${ }^{1}$ Laboratoire AIM, CEA/DSM-CNRS-Université Paris Diderot, IRFU/SAp Centre de Saclay, \\ F-91191 Gif-sur-Yvette, France \\ email: stephane.mathis@cea.fr \\ ${ }^{2}$ Observatoire de Paris-LESIA 5, place Jules Janssen, F-92195 Meudon Cedex \\ ${ }^{3}$ Argelander Institut für Astronomie, Universität Bonn, Auf dem Hügel 71, D-53111 Bonn, \\ Germany \\ email: vduez@astro.uni-bonn.de; jonathan@astro.uni-bonn.de
}

\begin{abstract}
The understanding of fossil fields origin, topology, and stability is one of the corner stones of the stellar magnetism theory. On one hand, since they survive on secular time scales, they may modify the structure and the evolution of their host stars. On the other hand, they must have a complex stable structure since it has been demonstrated that the simplest purely poloidal or toroidal fields are unstable on dynamical time scales. In this context, the only stable stellar configurations found today are those resulting from numerical simulations by Braithwaite and collaborators who studied the evolution of an initial stochastic magnetic field, which relaxes with a selective decay of magnetic helicity and energy, on mixed stable configurations (poloidal and toroidal) that seem to be in equilibrium and then diffuse. In this talk, we report the semianalytical investigation of such an equilibrium field in the axisymmetric case. We use variational methods, which describe selective decay of magnetic helicity and energy during MHD relaxation, and we identify a supplementary invariant due to the stable stratification of stellar radiation zones. This leads to states that generalize force-free Taylor's relaxation states studied in plasma laboratory experiments that become non force-free in the stellar case. Moreover, astrophysical applications are presented and the stability of obtained configurations is studied.
\end{abstract}

Keywords. magnetohydrodynamics (MHD), plasmas, stars: magnetic field

\section{Introduction}

Magnetic fields are now detected more and more often at the surface of main-sequence (and pre main-sequence) intermediate mass and massive stars, which have an external radiative envelope. Indeed, strong fields (300 $\mathrm{G}$ to $30 \mathrm{kG})$ are observed in some fraction of Herbig stars (Alecian et al. 2008), A stars (the Ap stars, see Aurière et al. 2007), as well as in B stars and in a handful of O stars (Grunhut et al. 2009). Furthermore, we cannot dismiss the possibility of a large-scale magnetic field being responsible for the quasi-uniform rotation of the bulk of the solar radiation zone, as revealed by $p$-modes helioseismology (Eff-Darwich et al. 2008). Finally, non convective compact objects display fields strength of $10^{4}-10^{9} \mathrm{G}$ for white dwarfs and of $10^{8}-10^{15} \mathrm{G}$ for neutron stars. Magnetic fields in stably stratified non convective stellar regions will thus be able to deeply modify our vision of stars evolution since their formation (Commerçon et al. 2010) to their late stages, for example for gravitational supernovae. Indeed, they will modify stellar internal dynamics, for example the transport of angular momentum and the resulting rotation history, and chemicals mixing (see Mathis \& Zahn 2005).

The large-scale, ordered nature (often approximately dipolar) of such magnetic fields and the scaling of their strengths as a function of their host properties (according to the 
flux conservation scenario) favour a fossil hypothesis, whose origin has to be understood. One of the fundamental question is thus the understanding of the topology of these largescale magnetic fields. To have survived since the star's formation or the PMS stage, a field must be stable on a dynamic (Alfvén) timescale. It was suggested by Prendergast (1956) that a stellar magnetic field in stable axisymmetric equilibrium must contain both poloidal (meridional) and toroidal (azimuthal) components, since both are unstable on their own (Tayler 1973; Wright 1973; Braithwaite 2006; Bonanno \& Urpin 2008b). This was confirmed recently by numerical simulations by Braithwaite \& Spruit (2004); Braithwaite \& Nordlund (2006); Braithwaite (2008) who showed that initial stochastic helical fields evolve on an Alfvén timescale into stable configurations: axisymmetric and nonaxisymmetric mixed poloidal-toroidal fields were found. This phenomenon well known in plasma physics is a MHD turbulent relaxation (i.e. self-organization process involving magnetic reconnections in resistive MHD). In this short paper, we present our physical understanding of such mechanism in stellar interiors focusing on the axisymmetric case. First, we show how to derive such magnetic configurations. Then, stability properties are studied. Astrophysical consequences and perspectives are finally discussed.

\section{The relaxed non force-free configuration}

In this work, we deal with axisymmetric, non force-free magnetic configurations (i.e. with a non-zero Lorentz force) in equilibrium inside stellar radiation zones, which result from an initial MHD relaxation of the field created by a PMS dynamo or the stellar formation. We first restrict ourselves to the non-rotating case, but results also apply to radiation regions in a state where rotation is uniform (Woltjer 1959), that could be the case if magnetic field is strong enough, and where meridional circulation can be neglected (i.e. if the star is near an equilibrium where the Lorentz torque vanishes, does not loose angular momentum, and have a stationary structure: see Mestel, Moss \& Tayler 1988; Busse 1981; Zahn 1992; Decressin et al. 2009). The more general case including differential rotation (and induced meridional circulation) will be treated in a near future.

Several reasons inclined us to focus on non force-free relaxed equilibria instead of forcefree ones, which are often studied in plasma laboratory experiments. First, Reisenegger (2009) reminds us that no configuration can be force-free everywhere. Although there do exist "force-free" configurations, they must be confined by some region or boundary layer with non-zero or singular Lorentz force. This induces discontinuities such as current sheets, which are unlikely to appear in nature except in a transient manner. Second, non force-free equilibria have been identified in plasma physics as the result of MHD relaxation (see for example Montgomery \& Phillips 1988; Shaikh et al. 2008). Third, as shown by Duez \& Mathis (2010), this family of equilibria is a generalization of Taylor states (force-free relaxed equilibria in plasma laboratory experiments; see Taylor 1974) in a stellar context, where the stable stratification of the medium plays a crucial role.

\subsection{The magnetic field in MHS equilibrium}

Let us describe the assumptions made in building the semi-analytical model of relaxed magnetohydrostatic (MHS) equilibrium described by Duez \& Mathis (2010). The axisymmetric magnetic field $\boldsymbol{B}(r, \theta)$ is expressed as a function of a poloidal flux $\Psi(r, \theta)$, a toroidal potential $F(r, \theta)$, and the potential vector $\boldsymbol{A}(r, \theta)$ so that it is divergence-free by construction:

$$
\boldsymbol{B}=\frac{1}{r \sin \theta}\left(\nabla \Psi \times \hat{\mathbf{e}}_{\varphi}+F \hat{\mathbf{e}}_{\varphi}\right)=\nabla \times \boldsymbol{A}
$$


where in spherical coordinates the poloidal component $\left(\boldsymbol{B}_{\mathrm{P}}\right)$ is in the meridional plane $\left(\hat{\mathbf{e}}_{r}, \hat{\mathbf{e}}_{\theta}\right)$ and the toroidal component $\left(\boldsymbol{B}_{\mathrm{T}}\right)$ is along the azimuthal direction $\left(\hat{\mathbf{e}}_{\varphi}\right)$. The MHS equation expressing balance between the pressure gradient force, gravity and the Lorentz force is

$$
\boldsymbol{O}=-\nabla P-\rho \nabla V+\frac{1}{\mu_{0}}(\nabla \times \boldsymbol{B}) \times \boldsymbol{B},
$$

where $V$ is the gravitational potential, which satisfies the Poisson equation: $\nabla^{2} V=4 \pi G \rho$.

\subsection{The non force-free relaxed equilibria family}

\subsubsection{MHD relaxation and variational method}

Here, we focus on the minimum energy non force-free MHS equilibrium that a stably stratified radiation zone can reach. First, given the field strengths in real stars, the ratio of the Lorentz force to gravity is very low: stellar interiors are thus in a regime where $\beta=P / P_{\mathrm{Mag}} \gg 1, P_{\mathrm{Mag}}=B^{2} /\left(2 \mu_{0}\right)$ being the magnetic pressure. Then, we identify the invariants governing the evolution of the reconnection phase, that leads to relaxed states in the non force-free case. The first one is the magnetic helicity

$$
\mathcal{H}=\int_{\mathcal{V}} \boldsymbol{A} \cdot \boldsymbol{B} \mathrm{d} \mathcal{V}
$$

which is an ideal MHD invariant known to be roughly conserved at large scales during relaxation. The second one is the mass encompassed in poloidal magnetic surfaces

$$
M_{\Psi}=\int_{\mathcal{V}} \Psi \rho \mathrm{d} \mathcal{V}
$$

conserved because of the stable stratification, which inhibits the radial movements and thus the transport of mass and flux in this direction. Note that this invariant can also be seen as a topological constraint. (see Moffatt 1985). Next, we assume a selective decay during relaxation (c.f. Biskamp 1997), in which the magnetic energy $E_{\text {mag }}=\int_{\mathcal{V}} \frac{\mathbf{B}^{2}}{2 \mu_{0}} \mathrm{~d} \mathcal{V}$ ( $\mu_{0}$ being the vaccum magnetic permeability), and thus the total energy

$$
E=E_{\text {mag }}+\frac{1}{2} \int_{\mathcal{V}} \rho(V+2 \mathcal{U}) \mathrm{d} \mathcal{V}
$$

where $\mathcal{U}$ is the specific internal energy per unit mass, decays much faster than $\mathcal{H}$ and $M_{\Psi}$, so that they can be considered constant on an energetic decay e-folding time. This is due to the stable stratification and to the different orders of spatial derivatives involved in the variation of $E_{\text {mag }}$ and $\mathcal{H}$ :

$$
\frac{\mathrm{d} E_{\mathrm{mag}}}{\mathrm{d} t}=-\int_{\mathcal{V}} \eta \boldsymbol{j}^{2} \mathrm{~d} \mathcal{V} \text { and } \frac{\mathrm{d} \mathcal{H}}{\mathrm{d} t}=-\int_{\mathcal{V}} \eta \boldsymbol{j} \cdot \boldsymbol{B} \mathrm{d} \mathcal{V},
$$

where $\eta$ is the magnetic diffusivity and $\mu_{0} \boldsymbol{j}=\boldsymbol{\nabla} \times \boldsymbol{B}, \boldsymbol{j}$ being the current. The reached equilibrium is thus the one of minimum energy for given magnetic helicity and mass encompassed in magnetic flux tubes. This can be determined applying a variational method where we minimize $E$ with respect to $\mathcal{H}$ and $M_{\Psi}$

$$
\delta E+a_{\mathcal{H}} \mathcal{H}+a_{M_{\Psi}} M_{\Psi}=0
$$

where $a_{\mathcal{H}}$ and $a_{M_{\Psi}}$ are Lagrangian multipliers. This allows to derive the elliptic linear partial differential equation governing $\Psi$ (Woltjer 1959; Montgomery \& Phillips 1989; Duez \& Mathis 2010):

$$
\Delta^{*} \Psi+\frac{\lambda_{1}^{2}}{R^{2}} \Psi=-\mu_{0} \bar{\rho} r^{2} \sin ^{2} \theta \beta_{0}
$$


Here, $\bar{\rho}$ is the density in the non-magnetic case, $\Delta^{*} \Psi \equiv \partial_{r r} \Psi+\sin \theta \partial_{\theta}\left(\partial_{\theta} \Psi / \sin \theta\right) / r^{2}$ the Grad-Shafranov operator in spherical coordinates, $\lambda_{1}$ the eigenvalue to be determined, $R$ a characteristic radius, and $\beta_{0}$ is constrained by the field's intensity. We have identified $a_{\mathcal{H}}=-\frac{1}{\mu_{0}} \frac{\lambda_{1}}{R}$ and $a_{M_{\Psi}}=-\beta_{0}$. Note that if $M_{\Psi}$ is not taken into account, we recover force-free minimum magnetic energy equilibria for a given helicity derived by Taylor (1974).

This equation is similar to the Grad-Shafranov equation used to find MHS equilibria in magnetically confined plasmas (Grad \& Rubin 1958; Shafranov 1966), the source term being here related to the stellar structure through $\bar{\rho}$ (for a discussion of the general form of this equation in astrophysics, see Heinemann \& Olbert 1978). Furthermore, this equilibrium is in a barotropic state (in the hydrodynamic meaning of the term, i.e. isobar and iso-density surfaces coincide) where the field is explicitly coupled with stellar structure through $\boldsymbol{\nabla} \times\left(\boldsymbol{F}_{\mathcal{L}} / \bar{\rho}\right)=\boldsymbol{O}$, where $\boldsymbol{F}_{\mathcal{L}}$ is the Lorentz force. This is a generalization of Prendergast's equilibrium taking into account compressibility, first studied in polytropic cases by Woltjer (1960).

\subsubsection{Solution}

The boundary conditions have now to be discussed. In Duez, Mathis \& Turck-Chièze (2010) and Duez \& Mathis (2010), we considered the general case of a field confined between two radii, owing to the possible presence of both a convective core and a convective envelope and to ensure the conservation of magnetic helicity. We here choose to cancel both radial and latitudinal fields at the surface, to avoid any current sheets, conserving once again magnetic helicity; the possible effects of the convective core on the large-scale surrounding field are neglected. Using Green's function method we finally obtain the purely dipolar, general solutions indexed by $i$ :

$$
\begin{aligned}
\Psi_{i}(r, \theta)= & -\mu_{0} \beta_{0} \frac{\lambda_{1}^{i}}{R} r\left\{j_{1}\left(\lambda_{1}^{i} \frac{r}{R}\right) \int_{r}^{R}\left[y_{1}\left(\lambda_{1}^{i} \frac{\xi}{R}\right) \bar{\rho} \xi^{3}\right] \mathrm{d} \xi\right. \\
& \left.+y_{1}\left(\lambda_{1}^{i} \frac{r}{R}\right) \int_{0}^{r}\left[j_{1}\left(\lambda_{1}^{i} \frac{\xi}{R}\right) \bar{\rho} \xi^{3}\right] \mathrm{d} \xi\right\} \sin ^{2} \theta,
\end{aligned}
$$

$R$ being the upper boundary confining the magnetic field; $\lambda_{1}^{i}$ are the set of eigenvalues indexed by $i$ allowing to verify the boundary conditions. The functions $j_{l}$ and $y_{l}$ are respectively the spherical Bessel functions of the first and the second kind.

As shown in Duez \& Mathis (2010), the first radial mode is the lowest energy state for given $\mathcal{H}$ and $M_{\Psi}$; we thus focus here only on this mode $i=1$. The toroidal magnetic field is then given using $F(\Psi)=\lambda_{1}^{1} \Psi / R$. Furthermore, this state is ruled by the following helicity-energy relation

$$
\mathcal{H}=\frac{2 \mu_{0} R}{\lambda_{1}^{1}}\left(E_{\text {mag }}-\frac{1}{2} \beta_{0} M_{\Psi}\right),
$$

which generalizes the one known in plasma physics for Taylor states to the stellar non force-free case.

In the case of a stably stratified $n=3$ polytrope (a good approximation to an upper main-sequence star radiative envelope) where we set $R=0.85 R_{*}$, we have $\lambda_{1}^{1} \simeq 32.95$ (represented in FIG. 1), while for a constant density profile, we have $\lambda_{1}^{1} \simeq 5.76$.

\subsubsection{Comparison with numerical simulations}

Let us now compare our analytical configuration to those obtained using numerical simulations (see Braithwaite \& Spruit 2004; Braithwaite \& Nordlund 2006; Braithwaite 

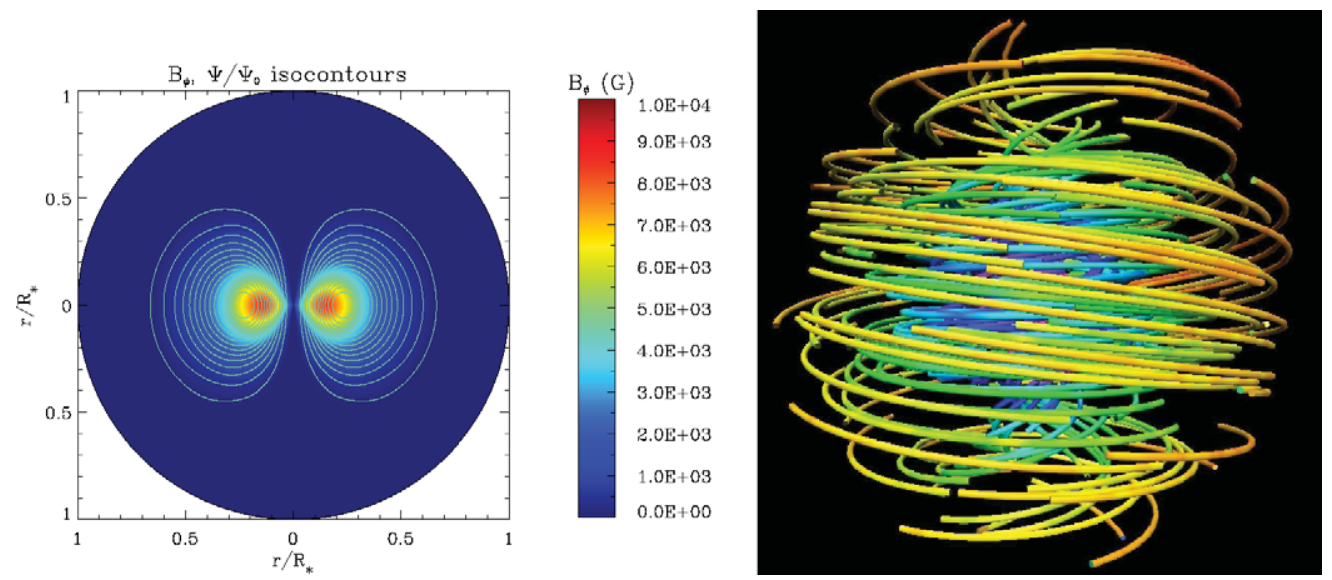

Figure 1. Left: toroidal magnetic field strength in colorscale (arbitrary field's strength) and normalized isocontours of the poloidal flux function $(\Psi)$ in meridional cut for the lowest energy equilibrium configuration $\left(\lambda_{1}^{1} \simeq 33\right.$ ); the neutral line is located at $r \simeq 0.23 R_{*}$. Right: magnetic field lines representing this mixed field configuration in 3-D looking from the side (the colorscale is a function of the density). Taken from Duez, Braithwaite \& Mathis (2010).

2008) in more details. Braithwaite and collaborators performed numerical magnetohydrodynamical simulations of the relaxation of an initially random magnetic field in a stably stratified star. Then, this initial magnetic field is always found to relax on the Alfvén time scale into a stable magneto-hydrostatic equilibrium mixed configuration consisting of twisted flux tube(s). Two families are then identified: in the first, the equilibria configurations are roughly axisymmetric with one flux tube forming a circle around the equator, such as in our configuration; in the second family, the relaxed fields are non-axisymmetric consisting of one or more flux tubes forming a complex structure with their axis lying at roughly constant depth under the surface of the star. Whether an axisymmetric or non-axisymmetric equilibrium forms depends on the initial condition chosen for the radial profile of the initial stochastic field strength $\|\mathbf{B}\| \propto \bar{\rho}^{p}$ : a centrally concentrated one evolves into an axisymmetric equilibrium as in our configuration while a more spreadout field with a stronger connection to the atmosphere relaxes into a non-axisymmetric one. Braithwaite (2008) indicates that, if using an ideal-gas star modeled initially with a polytrope of index $n=3$, the threshold is $p \approx 1 / 2$.

Moreover, as shown in Fig. 7 in Braithwaite (2008), the selective decay of the magnetic helicity $(\mathcal{H})$ and of the magnetic energy $\left(E_{\text {mag }}\right)$ assumed in $§ 2.1 .1$. occurs during the initial relaxation with a stronger decrease in $E_{\text {mag }}$ than that of $\mathcal{H}$. Furthermore, the transport of flux and mass in the radial direction is inhibited because of the stable stratification and the mass encompassed in poloidal magnetic surfaces is conserved (i.e. $M_{\Psi}$ ). The obtained configuration is of course non force-free.

Finally, note that our analytical configuration for which $E_{\mathrm{mag} ; \mathrm{P}} / E_{\mathrm{mag}} \approx 5.23 \times 10^{-2}$ (where $E_{\text {mag;P }}=\int_{\mathcal{V}} \boldsymbol{B}_{\mathrm{P}}^{2} /\left(2 \mu_{0}\right) \mathrm{d} \mathcal{V}$ ) verifies the stability criterion derived by Braithwaite (2009) for axisymmetric configurations: $\mathcal{A} E_{\mathrm{mag}} / E_{\text {grav }}<E_{\mathrm{mag} ; \mathrm{P}} / E_{\mathrm{mag}} \leqslant 0.8$, where $E_{\text {grav }}$ is the gravitational energy in the star, and $\mathcal{A}$ a dimensionless factor whose value is $\sim 10$ in a main-sequence star and $\sim 10^{3}$ in a neutron star, while we expect $E_{\text {mag }} / E_{\text {grav }}<10^{-6}$ in a realistic star (see for example Duez, Mathis \& Turck-Chièze 2010). Our analytical solution is thus similar to the axisymmetric non force-free relaxed solution family obtained by Braithwaite \& Spruit (2004) and Braithwaite \& Nordlund (2006). 
These configurations can thus be relevant to model initial equilibrium conditions for evolutionary calculations involving large-scale fossil fields in stellar radiation zones (see for example Mathis \& Zahn 2005; Brun \& Zahn 2006; Garaud \& Guervilly 2009).

\section{Stability: numerical method}

\subsection{The numerical model}

Analytical methods have been powerful to prove linear instabilities of magnetic configurations but unable to study nonlinear phases and to demonstrate stability. For this reason, we now turn on numerical simulations. The setup of the numerical model is similar to that in Braithwaite \& Nordlund (2006), where a fuller account can be found. We use the STAGGER code (Nordlund \& Galsgaard 1995), a high-order finite-difference Cartesian MHD code containing a "hyper-diffusion" scheme. The resolution is $192^{3}$. We model the star as a self-gravitating ball of ideal gas $(\gamma=5 / 3)$ with radial density and pressure profiles initially obeying the polytropic (thus barotropic) relation $P \propto \rho^{1+(1 / n)}$, with index $n=3$, which models an upper main-sequence star radiative envelope. It seems unlikely that a different EOS will make even much quantitative difference to the results; the important point is the stable stratification. We use this model to compare the dynamical evolution of the mixed (poloidal-toroidal) configuration to that of its purely poloidal and toroidal components on their own, both of which are unstable as mentioned above. We should therefore see these instabilities, growing on an Alfvén timescale. To test the stability of the configurations, we add a random "white noise" perturbation to the density field. The perturbation in density ( $1 \%$ in amplitude) contains length scales ranging from $R_{*}$ to $0.08 R_{*}$, the latter being double the Nyquist wavelength. This is roughly equivalent to azimuthal wavenumbers up to $m=38$ at a radius of $R_{*} / 2$.

\subsection{Results}

\subsubsection{Purely poloidal component}

The simulation is run for around ten Alfvén crossing times $\tau_{\mathrm{A}}$, over which time the instability grows, becomes nonlinear and results in the destruction of most of the original magnetic energy. The magnetic field amplitude is plotted at the left of FIG. 2, split into components according to azimuthal wavenumber $m$; obviously at $t=0$ all the energy is in the axisymmetric $m=0$ part. Note the clear transition at $t \approx 2 \tau_{\mathrm{A}}$ from the linear phase to the nonlinear, reconnective phase.
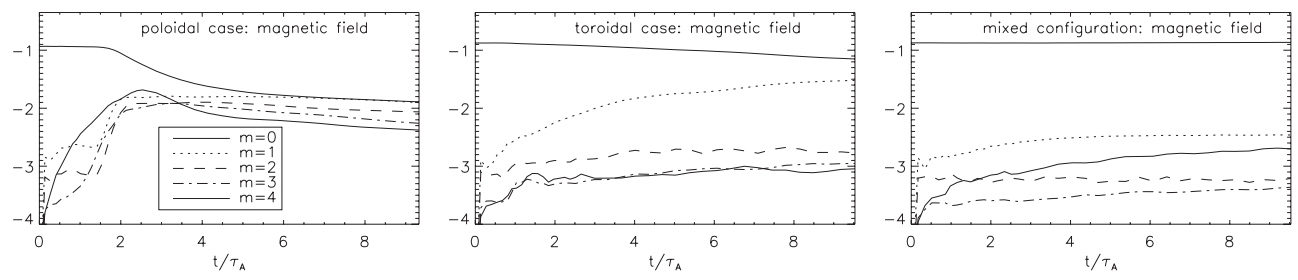

Figure 2. Time evolution of the (log) amplitudes in azimuthal modes $m=0$ to 4 averaged over the stellar volume of the magnetic field in the simulations with the purely poloidal field (left), purely toroidal field (middle) and the mixed field (right). Initially, all the magnetic energy is in the $m=0$ mode since the initial conditions are axisymmetric. Taken from Duez, Braithwaite \& Mathis (2010). 


\subsubsection{Purely toroidal component}

The middle plate of Fig. 2 shows the evolution of the toroidal field; clearly, the $m=$ 1 mode of the Tayler instability is dominant. After its linear growth, the instability manifests itself in the nonlinear regime (cf. Brun 2007; Elstner et al. 2008) mainly in the movement of spherical shells relative to one another. We expect eventual complete destruction of the field on a longer timescale.

\subsubsection{Mixed configuration}

The mixed poloidal-toroidal configuration exhibits completely different behaviour. The magnetic amplitudes are plotted on the right of FIG. 2, where we see an absence of growing modes. The kinetic energy present results simply from the initial perturbation and the oscillations and waves it sets up. No significant change in the configuration during the evolution is seen. To better examine the potentially unstable regions, we use Tayler's stability criteria (Tayler 1973) for purely toroidal fields and estimate the stabilisation from the poloidal component, following Braithwaite (2009). In FiG. 3, we plot this criteria for modes $m=0$ and $m=1$. The $m=0$ mode is unstable almost everywhere and the $m=1$ mode is unstable in a large cone around the poles; however the poloidal field stabilises these modes in most of the meridional plane except near the equatorial plane where it merely stabilises all wavelengths small enough to fit into the available space. Moreover, we can examine closely the behaviour of the field in the vicinity of the magnetic axis, where it can be approximated as the addition of an axial and a toroidal field (cylindrical geometry). Bonanno \& Urpin (2008a) outlined that in this case magnetic configurations can be subject to non-axisymmetric resonant instability. They determined the dependency of the Tayler instability maximum growth rate as a function of the azimuthal wave-number $m$ and of the ratio $\varepsilon$ of the axial field to the toroidal one. In our case, close to the center the flux function exhibits a behaviour in $\Psi \propto r^{2}$, so the azimuthal field is proportional to $s=r \sin \theta$ corresponding to the Bonanno et al.'s parameter $\alpha=1$. As underlined by the authors, in that case the maximum growth rate changes remarkably slowly with $m$ for all modes with $m \geqslant 2$ and the instability is weakly non-anisotropic. If we take as a value for $s_{1}$ the radius of the neutral line or the one where the azimuthal field is strongest, we obtain respectively $\varepsilon=0.64$ or $\varepsilon=0.79$. According to their study (see Bonanno \& Urpin 2008a, FIG. 7), we fulfill the stability criterion for the modes $m=0,1$ and 2 . Our results are therefore in agreement with their linear analysis.

\section{Conclusion and perspectives}

Using semi-analytic methods, we derived (with an appropriate choice of boundary conditions) then tested an axisymmetric non force-free relaxed magnetostatic equilibrium, which could exist in any non-convective stellar region: the radiative core of solar-type stars, the external envelope of massive stars, and compact objects. Using numerical simulations, we demonstrate the ability of the set-up to recover well-known instabilities in purely poloidal and toroidal cases, then find stability of the mixed configuration under all imaginable perturbations. We show the agreement of the result with linear analysis, highlighting the stabilizing influence of the poloidal field on the toroidal one, especially in the region close to the symmetry axis where purely toroidal fields usually develop kink-type instabilities in priority. This is the first time that the stability of an analytically-derived stellar magnetic configuration has been confirmed numerically (Duez, Braithwaite \& Mathis 2010).

This result has strong astrophysical implications: the configuration, as described in Duez \& Mathis (2010), provides a good initial condition to magneto-rotational transport 


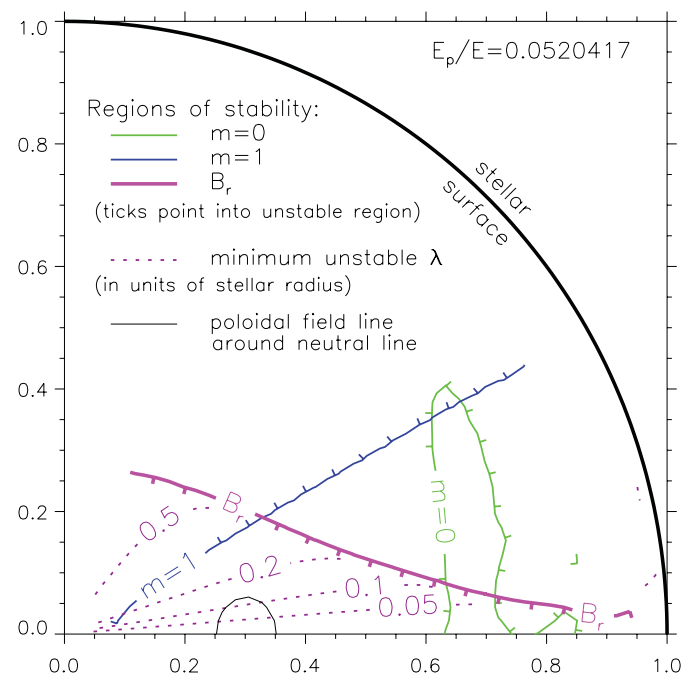

Figure 3. Half of the meridional plane, showing the regions stable against the $m=0$ and 1 Tayler modes in the absence of the poloidal component, and their stabilisation by the radial component $B_{r}$. Taken from Duez, Braithwaite \& Mathis (2010).

to be included in next generation stellar evolution codes and to magnetohydrodynamic simulations - where up to now the initial field would have been chosen arbitrarily; furthermore it will help to appreciate the internal magnetic structure of neutron stars, and various astrophysical processes involving magnetars.

Finally, we have to take into account differential rotation and induced meridional circulation in MHD relaxation theory in a near future.

Acknowledgments: S. Mathis thanks the organizers for this very nice conference. He also thanks A. Pouquet, K. Moffatt, J. Toomre, R. Arlt and A.-S. Brun for fruitful discussions. This work was supported in part by PNPS (CNRS/INSU).

\section{References}

Alecian, E., et al. 2008, A\&A A, 481, L99

Aurière, M., et al. 2007, A\&A A, 475, 1053

Biskamp, D. 1997, Nonlinear Magnetohydrodynamics (Cambridge, UK: Cambridge University Press)

Bonanno, A. \& Urpin, V. 2008a, A\& $A, 488,1$

一. 2008b, A\&A A77, 35

Braithwaite, J. 2006, A\&A, 449, 451

-. 2008, MNRAS, 386, 1947

-. 2009, MNRAS, 397, 763

Braithwaite, J. \& Spruit, H. C. 2004, Nature, 431, 819

Braithwaite, J. \& Nordlund, A. 2006, A\&A, 450, 1077

Brun, A. S. 2007, AN, 328, 1137

Brun, A. S. \& Zahn, J.-P. 2006, A\&SA, 457, 665

Busse, F. H. 1981, Geophysical and Astrophysical Fluid Dynamics, 17, 215

Commerçon, B., Hennebelle, P., Audit, E., Chabrier, G., \& Teyssier, R. 2010, A 6 A, 510, L3

Decressin, T., Mathis, S., Palacios, A., Siess, L., Talon, S., Charbonnel, C., \& Zahn, J.-P. 2009, $A \& A, 495,271$

Duez, V. \& Mathis, S. 2010, $A \mathscr{E} A$, 517, A58 
Duez, V., Braithwaite, J., \& Mathis, S. 2010, ApJ, accepted

Duez, V., Mathis, S., \& Turck-Chièze, S. 2010, MNRAS, 402, 271

Eff-Darwich, A., Korzennik, S. G., Jiménez-Reyes, S. J., \& García, R. A. 2008, ApJ, 679, 1636

Elstner, D., Bonanno, A., \& Rüdiger, G. 2008, A\&A, 329, 717

Grad, H. \& Rubin, H. 1958, Proceedings of the Second United Nations International Conference on the Peaceful Uses of Atomic Energy, Vol. 31, IAEA, Geneva, 190-197

Garaud, P. \& Guervilly, C. 2009, ApJ, 695, 799

Grunhut, J. H., et al. 2009, MNRAS, 400, L94

Heinemann, M. \& Olbert, S. 1978, Journal of Geophysical Research, 83, 2457

Mathis, S. \& Zahn, J.-P. 2005, A\&AA, 440, 653

Mestel, L., Moss, D., \& Tayler, R. J. 1988, MNRAS, 231, 873

Moffatt, H. K. 1985, J. Fluid Mechanics, 159, 359

Montgomery, D. \& Phillips, L. 1988, Phys. Rev. A, 38, 2953

Montgomery, D. \& Phillips, L. 1989, Physica D, 37, 215

Nordlund, A. \& Galsgaard, K. 1995, A 3D MHD code for Parallel Computers, Tech. rep., http://www.astro.ku.dk/ aake/papers/95.ps.gz

Prendergast, K. H. 1956, ApJ, 123, 498

Reisenegger, A. 2009, $A \mathscr{E} A$ A, 499, 557

Shafranov, V. D. 1966, Reviews of Plasma Physics, 2, 103

Shaikh, D., Dasgupta, B., Hu, Q., \& Zank, G. P. 2008, J. Plasma Physics, 75, 273

Spruit, H. C. 2002, A\& $A, 381,923$

Tayler, R. J. 1973, MNRAS, 161, 365

Taylor, J. B. 1974, Phys. Rev. Lett., 33, 1139

Woltjer, L. 1959, ApJ, 130, 405

-. 1960, ApJ, 131, 227

Wright, G. A. E. 1973, MNRAS, 162, 339

Zahn, J.-P. 1992, A\&A, 265, 115

\section{Discussion}

C. Forest: What are the boundary conditions on relaxation? Is there a mechanism for helicity injection or extraction at tachocline?

S. MATHIS: In this work, we consider initially confined relaxed magnetic configurations as a first step. These then open due to ohmic dissipation. The case of open boundary conditions should be treated in a near future.

J. ToOmRE: In your work, you don't take into account the differential rotation and the induced meridional circulation. What could be the modification of relaxed configurations if those are treated?

S. MATHIS: In this work, we first study non-rotating purely magnetic equilibria as Braithwaite and collaborators. The obtained states are inchanged by the presence of a uniform rotation. However, if there is a differential rotation, a meridional circulation will be induced that will modify the obtained relaxed configurations (see Mestel, Moss \& Tayler 1988). 